\title{
Seminal Vesicle Adherent to Rectal Wall Following Neoadjuvant Chemoradiotherapy: A Potential False-Positive Diagnostic Pitfall
}

Samia Liaquat

vCUhealth

Michael O. Idowu

Virginia Commonwealth University

Bryce Hatfield

Virginia Commonwealth University

Follow this and additional works at: https://scholarscompass.vcu.edu/gme_posters

Part of the Medicine and Health Sciences Commons

(c) The Author

\section{Downloaded from}

https://scholarscompass.vcu.edu/gme_posters/16

This Clinical Case Reports is brought to you for free and open access by the VCU Health at VCU Scholars Compass. It has been accepted for inclusion in Graduate Medical Education (GME) Resident and Fellow Research Day Posters by an authorized administrator of VCU Scholars Compass. For more information, please contact libcompass@vcu.edu. 


\section{Seminal Vesicle Adherent to Rectal Wall Following Neoadjuvant Chemoradiotherapy: A Potential False-Positive Diagnostic Pitfall.}

Samia Liaquat, MD, Michael O. Idowu, MD, and Bryce S. Hatfield, MD

\section{Abstract:}

The standard of care for stage T3 and stage T4 rectal adenocarcinomas involves neoadjuvant chemoradiotherapy followed by either low anterior resection or abdominopelvic resection. The presence of residual adenocarcinoma or

positive surgical margins provides useful prognostic information and can influence ongoing adjuvant therapy. Although uncommon, mimics of treated adenocarcinoma may be present in the surgical specimen. A high index of suspicion is

critical in avoiding potential false-positive pitfall, and the exclusion of mimics of treated adenocarcinoma is paramount to accurate diagnosis and treatment. Seminal vesicle epithelium has long been a challenge in differentiating prostatic

adenocarcinoma from benign epithelium. However, the role of incidental seminal vesiculectomy in recta resections due to fibrous adhesion to rectal wall secondary to chemoradiation has not been studied. As the seminal vesicle epithelium can show markedly atypical nuclei with radiation-type effect at baseline, the potential risk of misinterpretation as residual adenocarcinoma is high. In this article, we present 2 case reports of rectal adenocarcinoma treated with neoadjuvant chemoradiotherapy

followed by transabdominal resection (low anterior resection or abdominopelvic resection) with incidental seminal vesiculectomies mimicking either residual adenocarcinoma or residual

adenocarcinoma at a margin of resection.

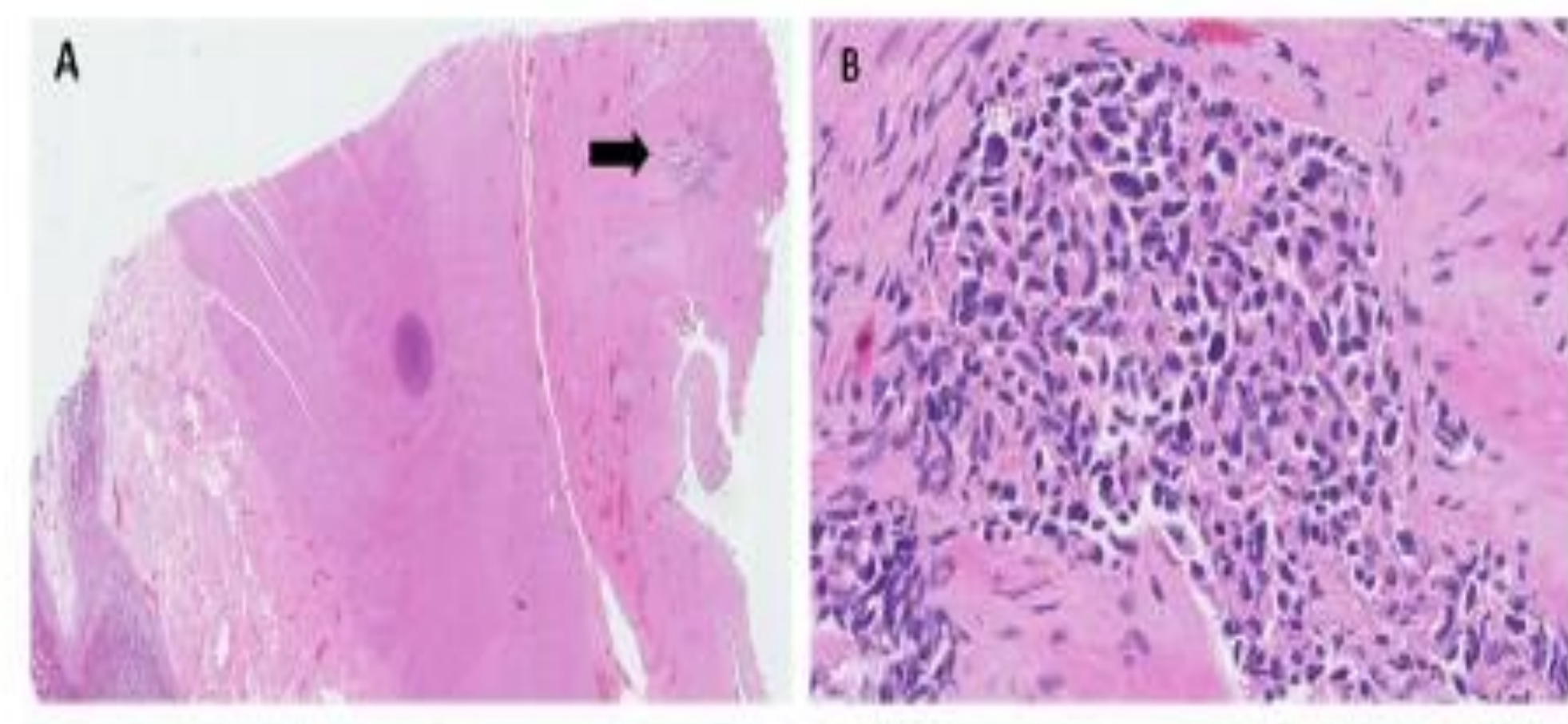

\section{Case \#1}

The first patient is a 63-year-old male with a history of prior LAR at an outside hospital for rectal adenocarcinoma reportedly
involving the rectosigmoid junction and invading into, but not involving the rectosigmoid junction and invading into, but not through, the muscularis propria (pT2) the previous year. A total of
12 lymph nodes were resected and reported as negative (pNO) 12 lymph nodes were resected and reported as negative ( $\mathrm{pNO}$ ), and all surgical margins were negative for carcinoma. Testing
mismatch repair proteins showed intact expression for MLH1, mismatch repair proteins showed intact expression for neoadjuvant or adjuvant CRT as a result of this initial diagnosis, Network guidelines for pT2 rectal carcinoma. Eleven months later, the patient presented with a 2-cm mass at the anastomotic site on surveillance colonoscopy. A biopsy of the mass demonstrated recurrent adenocarcinoma, and the patient was referred to our institution for surgical resection. Clinical restaging showed no evidence of metastatic disease, and the patient was treated with 6 weeks of preoperative 5-fluorouracil-based CRT a total dose of 50.4 Gy, followed by an APR. Grossly, no residua focus of atypical glandular tissue was noted in the wall of the rectum (Figure $1 \mathrm{~A}$, arrow). Microscopic examination at higher power revealed irregular, braching cleftlike glandular lumina outside the muscularis propria. The glandular cells contained atypical cells with dark nuclei and occasional intranuclear inclusions, and scant golden-brown pigment, surrounded by fibromuscular stroma (Figure 1B). Significant cautery artifact was present. On immunohistochemistry, the atypical glandular epithelium was negative for CDX2 (Figure 1C) and CK20 (Figure 1D), and positive for CD10 (Figure 1E) and p63 (Figure 1F). were consistent with seminal vesicle epithelium rather than residual adenocarcinoma

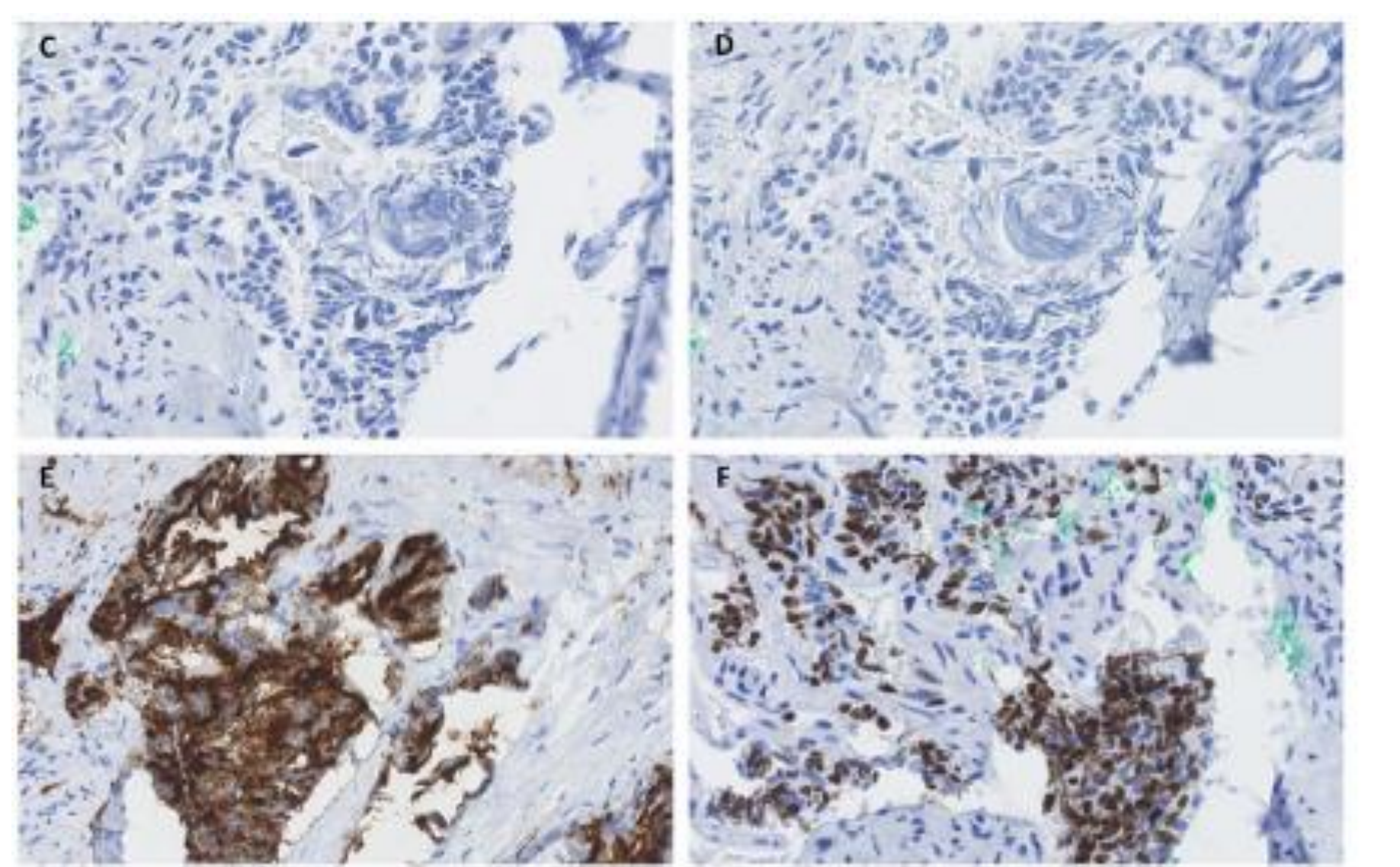

Figure 1. (A) Seminal vesicle attached to the wall of the rectum (arrow; hematoxylin-eosin [H\&E], 20x). (B) High-power view showing the seminal vesicle glandular epithelium with enlarged, hyperchromatic nuclei and adjacent fibromuscular stroma (H\&E, 400x). There was no staining for (C) CDX2 (CDX2, 400x) or (D) CK20 (CK20, 400x). The seminal vesicle epithelium showed positive staining for (E) CD10 (CD10, 400x) and (F) p63 (p63;

\section{Case \#2}

The second case is a 60-year-old male who presented with rectal bleeding and an elevated CEA of $32.6 \mathrm{ng} / \mathrm{mL}$. A diagnostic

colonoscopy revealed a $3 \mathrm{~cm}$ circumferential, ulcerated rectal mass $4 \mathrm{~cm}$ from anal verge. A biopsy showed moderately differentiated rectal adenocarcinoma. Clinical staging did not reveal any evidence of metastatic disease. On magnetic resonance imaging, the rectal mass penetrated $<5 \mathrm{~mm}$ beyond the muscularis propria with no definite solid organ invasion and an intact pertioneal reflection. The palient underwent eoadjuvant CRT and LAR. Grossly, a full-thickness ulcerated lesion measuring $1.3 \times 0.6 \times 0.5 \mathrm{~cm}$ was noted in the rectum. in perate focus of glandular tissue with irregular lumina pleomorphic nuclei, prominent nucleoli, and yellow cytoplasmic pigmentation/lipofuscin surrounded by a fibromuscular stroma (Figure 2A and B). The morphology was compatible with semin vesicle tissue, and the margins were reported as negative.

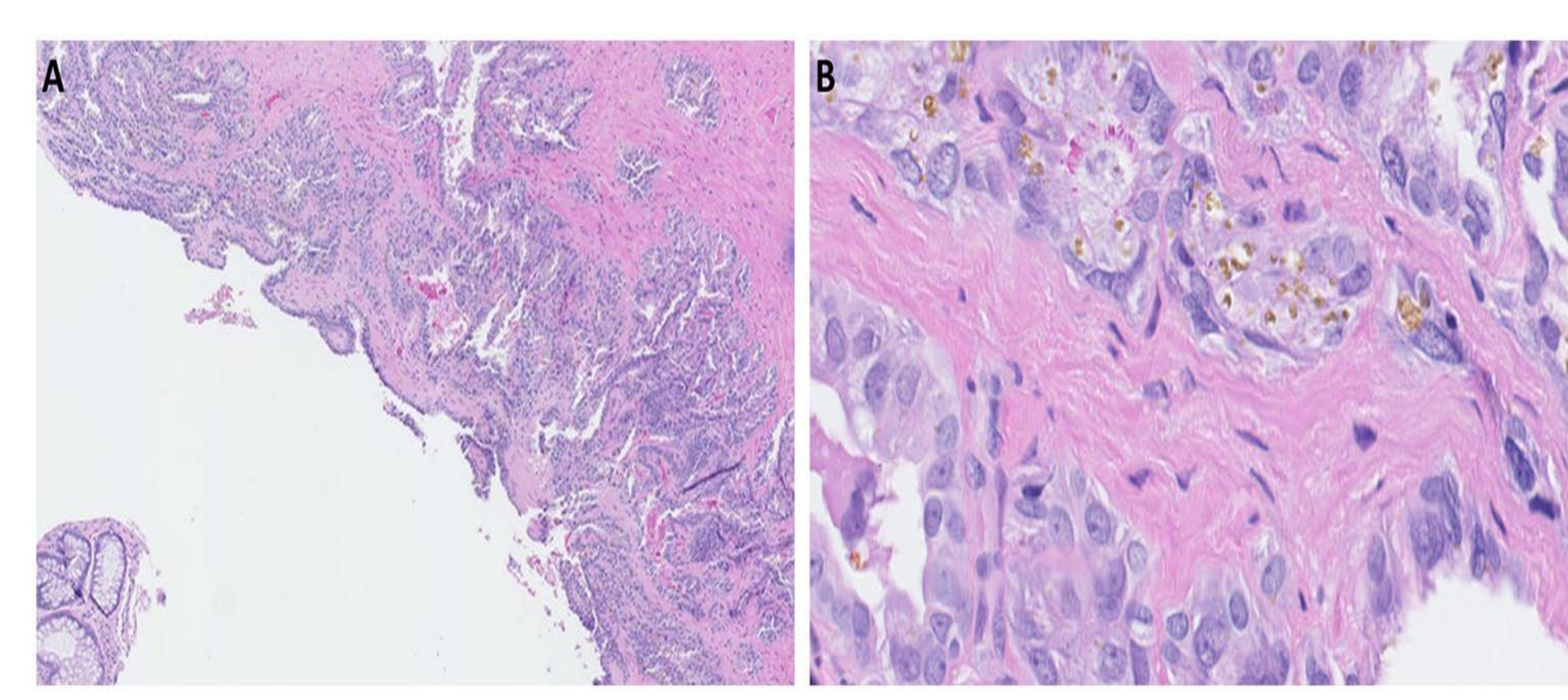

Figure 2. The second case contained a portion of seminal vesicle in the anastomotic donut. (A) At low-power view

(hematoxylineosin $[H \& E], 40 \times)$, the seminal vesicle epithelium and surrounding fibromuscular stroma is evident. (B) High-power magnification (H\&E, 400x) demonstrates well-formed glands wi cytoplasmic golden-brown pigment. The nuclei are large and

\section{Conclusion}

Although the incidence of incidental seminal vesiculectomy in rectal adenocarcinoma excisions treated with CRT is unknown, one should be aware of the possibility in order to discern it from residual tumor. Misdiagnosis of seminal

vesicle epithelium in case \#1 as adenocarcinoma could have led to the interpretation as partial response as opposed to pathologic complete response (ypT4a), potentially altering the patient's adjuvant therapy. Similarly, in case \#2,

diagnosis of seminal vesicle as residual adenocarcinoma at the anastomotic site may have led to misguided subsequent surgical re-excision of the margin or additional treatment. In order not to misinterpret seminal vesicle epithelium as

residual adenocarcinoma, careful attention to histologic features is paramount. Seminal vesicle epithelium can often be identified using histomorphologic features alone. In equivocal cases, staining for CDX2 and PAX8 can aid in differentiation. 